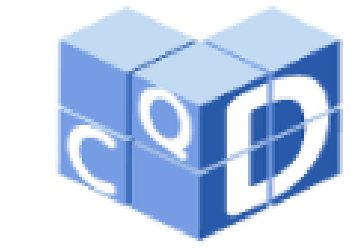

Revista Eletrônica

Paulista de Matemática ISSN 2316-9664

Volume 17, fev. 2020 Edição Ermac

Ariane Luzia dos Santos UNESP - Universidade Estadual Paulista "Júlio de Mesquita Filho"

ariane.santos@unesp.br

\section{Um estudo sobre desempenho em matemática dos alunos matriculados na rede estadual de ensino em uma avaliação em larga escala}

A study on mathematics performance of students enrolled in the state school system in a large-scale assessment

\begin{abstract}
Resumo
Este trabalho visa apresentar o desempenho médio em Matemática dos estudantes matriculados no $9^{\circ}$ ano do Ensino Fundamental na rede estadual de ensino, de acordo com o Sistema de Avaliação da Educação Básica (Saeb). Esta pesquisa quali-quantitativa tem como propósito estudar as notas em Matemática dos alunos registrados nas escolas estaduais das regiões brasileiras, conforme as competências e habilidades matemáticas descritas na escala de proficiência do Saeb. Para atingir a finalidade proposta, foram utilizados dados do Saeb referentes ao período entre 2007 a 2017. Os resultados indicam que há uma desigualdade na aprendizagem entre regiões brasileiras e um atraso nas proficiências matemáticas apresentadas pelos estudantes brasileiros analisados. Além disso, foi considerada uma categorização de aprendizagem que sugere um cenário de aprendizado insuficiente em Matemática por parte dos alunos analisados.
\end{abstract}

Palavras-chave: Ensino Fundamental. Avaliação externa. Proficiência em Matemática. Saeb.

\begin{abstract}
This paper aims to present the average performance in Mathematics of students enrolled in the 9th grade of elementary school in the state school system, according to the Basic Education Assessment System (Saeb). This qualitative and quantitative research aims to study the mathematical grades of students registered in state schools in the Brazilian regions, according to the skills and mathematical skills described in the Saeb proficiency scale. To achieve the proposed purpose, Saeb data were used for the period from 2007 to 2017 . The results indicate that there is a inequality in learning between Brazilian regions and a delay in the mathematical proficiency presented by the Brazilian students analyzed. In addition, it was considered a learning categorization that suggests a scenario of insufficient learning in Mathematics by the students analyzed.
\end{abstract}

Keywords: Elementary school. External evaluations. Proficiency in Mathematics. Saeb. 


\section{Introdução}

As avaliações externas em larga escala estão sendo muito utilizadas nas últimas três décadas para avaliar o sistema educacional brasileiro. O Sistema de Avaliação da Educação Básica (Saeb) visa analisar a condição do ensino fornecido nas escolas, gerando informações sobre os níveis de aprendizagem em Matemática, produzindo resultados para cada unidade escolar participante e para as redes de ensino. De acordo com o Instituto Nacional de Estudos e Pesquisas Educacionais Anísio Teixeira (INEP), autarquia federal responsável pelo Saeb e vinculado ao Ministério da Educação, os dados apresentados podem auxiliar no planejamento do trabalho pedagógico da escola e ser subsídio para a formulação e implementação de políticas públicas educacionais com a finalidade de melhorar a qualidade da Educação Básica.

As avaliações do Saeb são elaboradas a partir de matrizes de referência, que descrevem os conteúdos associados a habilidades e competências a serem analisadas em cada ano escolar considerado. As matrizes de referência do Saeb foram subdivididas em partes menores, cada uma especificando o que os itens das provas devem aferir, chamadas de descritores.

Os descritores retratam uma relação entre os conteúdos curriculares e as competências e habilidades cognitivas desenvolvidas pelos alunos. A matriz de referência em Matemática do $9^{\circ}$ ano do Ensino Fundamental possui 37 descritores. Vale ressaltar que as matrizes de referência do Saeb não englobam todo o currículo escolar e não devem ser equiparadas com técnicas, estratégias de ensino ou encaminhamentos metodológicos, visto que as matrizes de referências abordam apenas habilidades que podem ser analisadas por meio desse tipo de instrumento de avaliação.

A metodologia empregada na elaboração das provas do Saeb é a Teoria de Resposta ao Item (TRI), cuja preocupação é com a análise de cada item e não com a prova inteira. Uma das características fundamentais dessa técnica de avaliação é que ela possibilita comparar desempenhos de alunos e acompanhar a evolução do sistema educacional brasileiro.

Neste contexto estamos interessados em analisar os dados fornecidos pelo Saeb sobre o desempenho médio em Matemática dos estudantes brasileiros matriculados no $9^{\circ}$ ano do Ensino Fundamental na rede estadual de ensino, conforme as competências e habilidades matemáticas descritas na escala de proficiência do Saeb. Será que há um atraso nas proficiências matemáticas apresentadas pelos estudantes brasileiros considerados? O objetivo é analisar as diferenças na aprendizagem em Matemática entre os estudantes das diversas regiões brasileiras, apreciando as cinco edições do Saeb na última década.

Para alcançar a finalidade proposta, inicialmente, foi realizada uma análise da matriz de referência e da escala de proficiência do Saeb, ambas são encontradas no site do INEP, e uma apreciação de artigos científicos que abordam esse tipo de avaliação e estão citados neste texto. Posteriormente, foi feito um estudo quali-quantitativo sobre os dados do desempenho dos alunos considerados na pesquisa, nas edições do Saeb ocorridas no período entre 2007 a 2017. Almeja-se que este estudo contribua para o debate sobre novas formas de intervenção na re- 
cuperação de componentes de aprendizagem em Matemática e nos processos de ensino e aprendizagem.

Parte desse trabalho foi apresentado no VI Encontro Regional de Matemática Aplicada e Computacional (ERMAC). Aqui foram acrescidas mais detalhes a respeito das reflexões sobre as descrições das habilidades e competências matemáticas que os estudantes possivelmente estão aptos de realizar em cada nível da escala de proficiência em Matemática do Saeb. Além disso, foi considerada uma categorização de aprendizagem em Matemática, conforme os níveis da escala de proficiência do Saeb.

O trabalho está dividido em quatro seções, incluindo esta introdução. A segunda seção aborda sobre a matriz de referência e a escala de proficiência de Matemática do Saeb, referentes ao $9^{\circ}$ ano do Ensino Fundamental. A seguir, são apresentados os resultados da análise das notas de desempenho em Matemática dos estudantes apreciados, considerando os resultados nas diferentes regiões brasileiras. Por fim, a quarta parte apresenta as considerações finais da pesquisa.

\section{Matriz de referência e escala de proficiência do Saeb}

A matriz de referência do Saeb é utilizada para propor habilidades e competências a serem analisadas em cada ano escolar considerado e nortear a elaboração das questões da prova do Saeb. Além disso, serve como base para preparação da escala de proficiência que especifica o que os estudantes provavelmente são capazes de desempenhar na avaliação do Saeb.

As matrizes de referência de Matemática do Saeb estão subdivididas em temas e estes, por sua vez, em descritores. Cada descritor relaciona os conteúdos curriculares e as competências e habilidades desenvolvidas pelos estudantes. Segundo o INEP (BRASIL, 2019), "cada descritor dá origem a diferentes itens e, a partir das respostas dadas, verifica-se quais habilidades os alunos efetivamente desenvolveram."

Os quatro temas das matrizes de referência de Matemática do Saeb são espaço e forma (na área da Geometria); grandezas e medidas (na área da Aritmética, da Álgebra, Geometria e de outras áreas); números e operações/ álgebra e funções (na área da Aritmética e da Álgebra) e tratamento de informação (dados estatísticos, Probabilidade e Combinatória). Esses temas estão de acordo com os blocos de conteúdos estabelecidos nos Parâmetros Curriculares Nacionais (BRASIL, 1998, p. 50).

A partir das matrizes de referência, em 1997, surgiu a escala de proficiência do Saeb para Matemática. Esse fato, segundo Horta Neto (2007, p. 9) permitiu comparar os resultados adquiridos pela edição do Saeb ocorrida em 1995 e possibilitou fazer o mesmo nas edições seguintes.

A escala de proficiência de Matemática do Saeb do $9^{\circ}$ ano do Ensino Fundamental inicia-se no nível 1 (desempenho maior ou igual a 200 e menor que 225), depois a cada nível vai 
aumentando 25 pontos até atingir o nível 9 (desempenho maior ou igual a 400 ). Em cada nível são adicionadas competências e habilidades em Matemática, considerando os temas da Matriz de referência do Saeb.

Segundo o INEP (BRASIL, 2019), a ordem dos itens, por nível, está em conformidade com os temas e não com a complexidade da habilidade. Além disso, o intervalo de cada nível inclui o primeiro ponto e exclui o último ponto e na escala atual há níveis que não apreciam todos os quatros temas das Matrizes de referência. Ainda de acordo com o INEP, esse fato ocorre porque não existem itens âncora que contemplem esse nível.

Cabe observar que é importante estudar as notas de desempenho e o seu nível correspondente com o propósito de perceber o significado pedagógico desses dados. Santos e Tolentino-Neto (2015, p. 318) ressaltam que "é preciso analisar a descrição das competências correspondentes a cada nível, ou seja, uma interpretação pedagógica que subsidie modificações no processo do ensino".

\section{Análise dos dados}

Esta seção tem a finalidade de estudar o comportamento das médias de desempenho em Matemática dos estudantes matriculados no $9^{\circ}$ ano do Ensino Fundamental na rede estadual de ensino. Os dados considerados foram obtidos nas edições do Saeb que ocorreram entre 2007 e 2017.

O Saeb emprega na elaboração das provas a Teoria de Resposta ao Item (TRI). Para Klein (2009, p.127) a TRI utiliza "modelos matemáticos onde a probabilidade de resposta a um item é modelada como função da proficiência (habilidade) do aluno e de parâmetros que expressam certas propriedades dos itens." Neste caso, a probabilidade do aluno acertar cada item é diretamente proporcional à sua proficiência.

De acordo com as características da TRI, é possível confrontar os resultados do Saeb ao longo dos anos porque a metodologia usada garante a comparabilidade dos resultados. Segundo Klein (2009, p.127), "no SAEB, o uso de itens comuns entre séries e entre anos permite que os alunos de todas as séries e de todos os anos sejam postos em uma mesma escala de proficiência de modo que seus desempenhos possam ser comparados".

Na tabela a seguir é apresentado o desempenho médio em Matemática dos estudantes matriculados no $9^{\circ}$ ano do Ensino Fundamental na rede estadual de ensino nas edições do Saeb que ocorreram no período de 2007 a 2017. Os dados são fornecidos por regiões brasileiras e de forma nacional. 
Tabela I - Desempenho médio em Matemática.

\begin{tabular}{c|c|c|c|c|c|c}
\hline Região & Nota 2007 & Nota 2009 & Nota 2011 & Nota 2013 & Nota 2015 & Nota 2017 \\
\hline Norte & 232,82 & 233,69 & 236,56 & 235,37 & 239,90 & 241,39 \\
\hline Nordeste & 226,93 & 228,94 & 231,35 & 232,37 & 240,68 & 240,62 \\
\hline Sudeste & 244,57 & 246,62 & 248,25 & 248,05 & 253,08 & 252,91 \\
\hline Sul & 252,09 & 252,99 & 254,29 & 250,12 & 257,19 & 262,67 \\
\hline Centro-Oeste & 241,61 & 241,84 & 245,65 & 246,08 & 252,91 & 255,66 \\
\hline Brasil & 241,63 & 242,87 & 245,08 & 244,75 & 250,53 & 252,58 \\
\hline
\end{tabular}

Fonte INEP. Elaboração Própria.

Observando a Tabela 1, percebe-se que na década estudada, em geral, houve um pequeno crescimento nas notas de desempenho em Matemática em relação a cada edição anterior do Saeb. Isso sugere que ocorreu um pequeno avanço no desempenho médio em Matemática apresentado pelos estudantes ao longo de uma década no ano escolar considerado. Mesmo assim, é importante dizer que a análise é feita em uma escala de proficiência cujo último patamar é determinado pelo desempenho maior ou igual a 400 pontos.

Vale destacar que os dados estudados indicam que há uma desigualdade na aprendizagem entre as regiões brasileiras. No período avaliado, percebe-se que, em média, as notas dos estudantes das regiões norte e nordeste são inferiores à média nacional enquanto que nas outras regiões, geralmente, as notas estão acima da média nacional. Essa diferença pode estar relacionada, entre outros fatores, com características socioeconômicas de cada região, com os percentuais do indicador de adequação de formação de docente, como sugerem os dados do censo escolar fornecidos pelo INEP, e com o processo de formação continuada de professores.

$\mathrm{Na}$ escala de proficiência de Matemática do $9^{\circ}$ ano do Ensino Fundamental, o Saeb apresenta em nove níveis as habilidades e competências cognitivas que os estudantes do ano escolar considerado provavelmente são capazes de realizar. Com base nessa escala podemos associar a Tabela 1 à tabela a seguir, segundo o nível de proficiência em Matemática.

Tabela 2 - Nível de proficiência em Matemática.

\begin{tabular}{c|c|c|c|c|c|c}
\hline Região & Nível 2007 & Nível 2009 & Nível 2011 & Nível 2013 & Nível 2015 & Nível 2017 \\
\hline Norte & 2 & 2 & 2 & 2 & 2 & 2 \\
\hline Nordeste & 2 & 2 & 2 & 2 & 2 & 2 \\
\hline Sudeste & 2 & 2 & 2 & 2 & 3 & 3 \\
\hline Sul & 3 & 3 & 3 & 3 & 3 & 3 \\
\hline Centro-oeste & 2 & 2 & 2 & 2 & 3 & 3 \\
\hline Brasil & 2 & 2 & 2 & 2 & 3 & 3 \\
\hline
\end{tabular}

Fonte INEP. Elaboração Própria.

Analisando a Tabela 2, percebe-se que há uma década os estudantes brasileiros matriculados no $9^{\circ}$ ano do Ensino Fundamental na rede estadual, em média, estavam no nível 2 de 
proficiência em Matemática e atualmente estão no nível 3, em uma escala de proficiência com nove níveis. Ou seja, em dez anos ocorreu um avanço discreto, mas ainda precisamos melhorar, uma vez que o estudo é realizado em uma escala de proficiência cujo o último estágio é o nível 9.

Nota-se também que no período analisado, nas regiões norte e nordeste, os estudantes matriculados no $9^{\circ}$ ano do Ensino Fundamental na rede estadual, em média, permanecem no nível 2. O mesmo acontece com os estudantes na região sul que continuam no nível 3 de proficiência em Matemática. Enquanto nas regiões sudeste e centro-oeste, os estudantes possuíam, em média, o nível 2 e nas duas últimas edições do Saeb apresentaram o nível 3 de proficiência em Matemática.

É importante fazer uma análise pedagógica desses dados fornecidos pelo Saeb. Segundo Klein (2009, p.137), a interpretação da escala "significa escolher alguns pontos ou níveis da escala e descrever os conhecimentos e habilidades que os alunos demonstram possuir quando situados em torno desses pontos".

As escalas de proficiência do Saeb colocam em ordem os desempenhos dos alunos do nível mais baixo ao mais alto, em uma sequência de habilidades, fazendo com que exista uma continuidade entre o ponto inicial e o final.

$\mathrm{Na}$ escala de proficiência em Matemática do $9^{\circ}$ ano do Ensino Fundamental, o INEP não utilizou itens do ano escolar considerado que avaliam as habilidades do nível 0 . O Instituto chama atenção que os estudantes do $9^{\circ}$ ano do Ensino Fundamental que apresentam o desempenho em Matemática menor que 200 pontos necessitam de uma atenção especial porque não demonstram habilidades bem elementares que deveriam apresentar nessa etapa escolar.

No nível 1, onde o desempenho é maior ou igual a 200 e menor que 225 pontos, segundo o INEP (BRASIL, 2011), os estudantes possivelmente são capazes de identificar o maior ou menor número em um conjunto de números racionais, representados na forma decimal. No tratamento de informações, os alunos devem interpretar dados expostos em tabela e gráficos de colunas.

O quadro a seguir descreve as habilidades e competências em Matemática consideradas nos níveis 2 e 3 da escala de proficiência de Matemática do Saeb do $9^{\circ}$ ano do Ensino Fundamental que foram, em média, apresentados pelos estudantes no período analisado, de acordo com a Tabela 2 acima. 
Quadro 1 - Níveis 2 e 3 da escala de proficiência em Matemática do Saeb.

\begin{tabular}{|c|c|}
\hline $\begin{array}{c}\text { Nível 2 (Desempenho maior ou igual a } \\
225 \text { e menor que 250) }\end{array}$ & $\begin{array}{c}\text { Além das habilidades anteriormente citadas, os } \\
\text { estudantes provavelmente são capazes de: } \\
\text { Números e operações; Álgebra e funções } \\
\text { Reconhecer a fração que corresponde à relação } \\
\text { parte-todo entre uma figura e suas partes hachura- } \\
\text { das. } \\
\text { Associar um número racional que representa uma } \\
\text { quantia monetária, escrito por extenso, à sua re- } \\
\text { presentação decimal. } \\
\text { Determinar uma fração irredutível, equivalente a } \\
\text { uma fração dada, a parir da simplificação por três. } \\
\text { Tratamento de informações } \\
\text { Interpretar dados apresentados em gráfico de } \\
\text { linhas simples. } \\
\text { Associar dados apresentados em gráfico de colu- } \\
\text { nas a uma tabela. }\end{array}$ \\
\hline
\end{tabular}




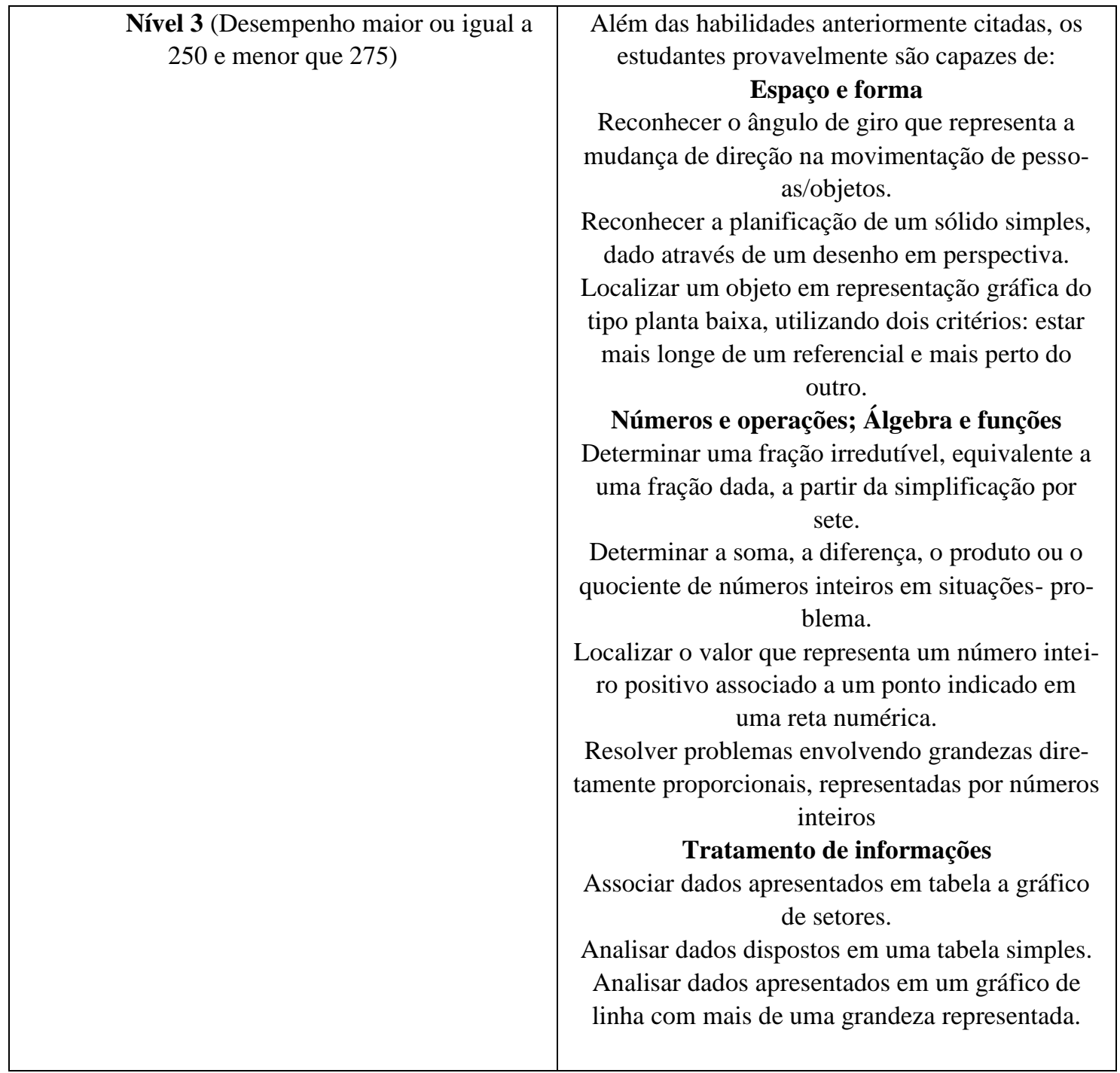

Fonte INEP.

De acordo com a Tabela 2, tem-se que, em média, os estudantes brasileiros matriculados no $9^{\circ}$ ano do Ensino Fundamental das escolas estaduais apresentam em média o nível 3 na escala de proficiência em Matemática do Saeb. Isso indica que os estudantes, de modo geral, não responderam aos itens que estão associados aos níveis mais elevados da escala de referência e não souberam trabalhar com elementos geométricos, algébricos, analíticos e estatísticos que se encontram nos últimos níveis da escala do Saeb.

Por exemplo, no nível 4, no qual o desempenho é maior ou igual a 275 e menor que 300, em média, os estudantes não conseguiram resolver problemas que era necessário transformar unidades de medidas de comprimento, de metros para centímetros ou resolver uma expressão algébrica de $1^{\circ}$ grau ou ainda, não analisaram dados distribuídos em uma tabela de dupla entrada.

No nível 5, no qual o desempenho maior ou igual a 300 e menor que 325, a titulo de exemplo, os estudantes não resolveram problema envolvendo grandezas diretamente proporcionais, definidas por números racionais na forma decimal, não localizaram dois ou mais pon- 
tos em um sistema de coordenadas e não conseguiram calcular o perímetro de uma região retangular ou o volume através da contagem de blocos.

Seguindo o mesmo raciocínio, no nível 6, no qual o desempenho é maior ou igual a 325 e menor que 350, em média, o aluno, não identificou frações equivalentes ou a relação entre as medidas de raio e diâmetro de uma circunferência. Além disso, não conseguiu resolver problemas usando o Teorema de Pitágoras no cálculo de medida da hipotenusa, conhecendo as medidas dos catetos ou encontrar o valor numérico de uma expressão algébrica que tenha parênteses, envolvendo números naturais.

Enquanto, no nível 7, no qual o desempenho é maior ou igual a 350 e menor que 375 , por exemplo, os estudantes não identificaram ângulos agudos, retos ou obtusos de acordo com sua medida em graus, não resolveram problemas envolvendo equação do $2^{\circ}$ grau, não analisaram dados distribuídos em uma tabela de três ou mais entradas.

No nível 8, no qual o desempenho é maior ou igual a 375 e menor que 400, por exemplo, os estudantes não resolveram problemas empregando as propriedades das cevianas (altura, mediana e bissetriz ) de um triângulo isósceles, com a ajuda da figura ou envolvendo grandezas inversamente proporcionais. Não calcularam a área de figuras simples (triângulo, paralelogramo, trapézio), inclusive usando composição/decomposição.

Finalmente, no nível 9, no qual o desempenho é maior ou igual a 400, por exemplo, os estudantes não resolveram problemas usando a soma das medidas dos ângulos internos de um polígono ou não reconheceram a expressão que indica uma regularidade existente em uma sequência de números ou de figuras geométricas.

Klein (2006, p.152) afirma que a interpretação da escala de proficiência do Saeb pode ser aproveitada para propor indicadores de qualidade do processo de aprendizagem do estudante. De acordo com o INEP (BRASIL, 2018, p.19), a secretaria de Educação Básica do ministério de educação - gestão 2018 fez uma classificação de aprendizagem por categoria, considerando a escala de proficiência em Matemática para o $9^{\circ}$ ano do Ensino Fundamental. A saber, insuficiente (nível 0 a 3), básico (nível 4 a 6) e adequado (nível 7 a 10).

Quadro 2 - Categoria de aprendizagem em Matemática.

\begin{tabular}{|c|c|c|c|c|c|c|}
\hline Região & Nível 2007 & Nível 2009 & Nível 2011 & Nível 2013 & Nível 2015 & Nível 2017 \\
\hline Norte & Insuficiente & Insuficiente & Insuficiente & Insuficiente & Insuficiente & Insuficiente \\
\hline Nordeste & Insuficiente & Insuficiente & Insuficiente & Insuficiente & Insuficiente & Insuficiente \\
\hline Sudeste & Insuficiente & Insuficiente & Insuficiente & Insuficiente & Insuficiente & Insuficiente \\
\hline Sul & Insuficiente & Insuficiente & Insuficiente & Insuficiente & Insuficiente & Insuficiente \\
\hline Centro-oeste & Insuficiente & Insuficiente & Insuficiente & Insuficiente & Insuficiente & Insuficiente \\
\hline Brasil & Insuficiente & Insuficiente & Insuficiente & Insuficiente & Insuficiente & Insuficiente \\
\hline
\end{tabular}

Fonte INEP. Elaboração Própria. 
Considerando essa categorização e o Quadro 2, observa-se que, em média, os estudantes brasileiros matriculados no $9^{\circ}$ ano do Ensino Fundamental na rede estadual de ensino apresentaram aprendizagem insuficiente no período analisado.

A análise dos dados do desempenho em Matemática divulgados pelo do Saeb associada à descrição dos níveis da escala de proficiência permite também estudar a relação entre o currículo oficial e o que está sendo desenvolvido nos processos de ensino e de aprendizagem. Nesse sentido, Castro (2000, p.126) afirma que o Saeb "releva a distância entre o currículo proposto e o currículo ensinado", porque esse instrumento de avaliação permite comparar os currículos propostos pelos governos e aquilo que está sendo efetivamente ensinado em sala de aula. Além disso, segundo Castro (2000, p.126), os resultados do Saeb possibilitam identificar as principais deficiências na aprendizagem dos estudantes.

Os dados divulgados pelo Saeb também podem auxiliar na proposta de políticas públicas educacionais e contribuir para que os docentes reflitam sobre suas ações no processo de ensino de Matemática e procurem novas metodologias de ensino. Klein (2006, p.152) sugere que "a interpretação da escala e as informações sobre os erros dos alunos deveriam ser utilizadas para fornecer subsídios para programas de formação e capacitação de professores”.

Além disso, as informações do Saeb precisam ser interpretadas e utilizadas pelo gestor da instituição de ensino de forma mais precisa. Klein (2006, p.155) afirma que esses dados evidenciam a necessidade de "rever as políticas de formação e capacitação dos professores".

Dessa forma, é importante avaliar os resultados do Saeb para verificar as competências cognitivas e habilidades em Matemática que não são desenvolvidas, em média, pelos estudantes brasileiros. Ademais, compete a cada gestor escolar fazer sua interpretação pedagógica e comparar seus resultados do Saeb com suas próprias metas traçadas. Castro (2000, p.127) acredita que a utilização dos resultados do Saeb permite "aos dirigentes das redes públicas desenvolverem um trabalho de formação continuada dos professores, com base nos resultados da avaliação da aprendizagem verificados".

\section{Conclusão}

Nas edições do Saeb ocorridas na última década, se constata um panorama insuficiente das habilidades e competências cognitivas requeridas para o aprendizado de Matemática nos estudantes brasileiros matriculados no $9^{\circ}$ ano do Ensino Fundamental na rede estadual de ensino, conforme a categorização de aprendizagem em Matemática considerada.

Em cada edição do Saeb, em geral, ocorreu um pequeno acréscimo nas notas em Matemática dos estudantes analisados. De qualquer forma, as médias de desempenho em Matemática estão distantes da maior nota na escala de proficiência em Matemática do Saeb considerada no estudo. 
Vale ressaltar que, usualmente, as médias de desempenho em Matemática desses estudantes nas regiões norte e nordeste foram menores que a média nacional enquanto nas outras regiões as médias ficaram acima da média nacional. Esse fato pode estar relacionado às características socioeconômicas de cada região e aos indicadores educacionais fornecidos pelo censo escolar realizado pelo INEP.

Esse cenário indica que com o aumento de habilidades e competência cognitivas matemáticas, há uma diminuição de associação por parte dos estudantes avaliados. Deste modo, em média, estes estudantes identificam as elementos matemáticos, mas não compreendem a situação-problema e não fazem uma correlação lógica para proporcionar uma resposta satisfatória do problema. Ademais, em média, há uma dificuldade para trabalhar com as componentes geométricas, algébricas, analíticas e estatísticas que se deparam nos últimos níveis da escala de proficiência em Matemática do Saeb.

Uma ampla divulgação, entre os gestores escolares e professores, do resultado do desempenho médio em Matemática dos estudantes no Saeb associado à interpretação da escala de proficiência de Matemática do Saeb permitirá uma proposição de novas formas de intervenção na recuperação de componentes de aprendizagem e na elaboração de ideias para formação continuada de professores. Consequentemente, existirá melhorias no processo de aprendizagem e no desempenho dos estudantes nas avaliações externas de larga escala.

\section{Referências}

BRASIL. Ministério da Educação. Parâmetros curriculares nacionais: matemática. Brasília: MEC, 1998.

INEP. Microdados - Saeb/prova Brasil. 2007a. Disponível em: http://portal.inep.gov.br/web/guest/microdados . Acesso em 10 set. 2018.

INEP. Resultados - Saeb/prova Brasil. 2007b. Disponível em: http://portal.inep.gov.br/web/guest/educacao-basica/saeb/resultados . Acesso em 10 set. 2018.

INEP. Microdados - Saeb/prova Brasil. 2009a. Disponível em: http://portal.inep.gov.br/web/guest/microdados . Acesso em 10 set. 2018.

INEP. Resultados - Saeb/prova Brasil. 2009b. Disponível em: http://portal.inep.gov.br/web/guest/educacao-basica/saeb/resultados . Acesso em 10 set. 2018.

INEP. Matrizes e escalas. 2011a. Disponível em: http://portal.inep.gov.br/web/guest/educacao-basica/saeb/matrizes-e-escalas . Acesso em 10 set. 2018 .

INEP. Microdados - Saeb/prova Brasil. 2011b. Disponível em: http://portal.inep.gov.br/web/guest/microdados . Acesso em 10 set. 2018. 
INEP. Resultados - Saeb/prova Brasil. 2011c. Disponível em:

http://portal.inep.gov.br/web/guest/educacao-basica/saeb/resultados . Acesso em 10 set. 2018.

INEP. Microdados - Saeb/prova Brasil. 2013a. Disponível em:

http://portal.inep.gov.br/web/guest/microdados . Acesso em 10 set. 2018.

INEP. Resultados - Saeb/prova Brasil. 2013b. Disponível em:

http://portal.inep.gov.br/web/guest/educacao-basica/saeb/resultados . Acesso em 10 set. 2018.

INEP. Microdados - Saeb/prova Brasil. 2015a. Disponível em:

http://portal.inep.gov.br/web/guest/microdados . Acesso em 10 set. 2018.

INEP. Resultados - Saeb/prova Brasil. 2015b. Disponível em:

http://portal.inep.gov.br/web/guest/educacao-basica/saeb/resultados . Acesso em 10 set. 2018.

INEP. Microdados - Saeb/prova Brasil. 2017a. Disponível em:

http://portal.inep.gov.br/web/guest/microdados . Acesso em 10 set. 2018.

INEP. Resultados - Saeb/prova Brasil. 2017b. Disponível em:

http://portal.inep.gov.br/web/guest/educacao-basica/saeb/resultados . Acesso em 10 set. 2018.

INEP. Press kit Saeb 2017. 2018. Disponível em:

http://download.inep.gov.br/educacao_basica/saeb/2018/documentos/presskit_saeb2017.pdf . Acesso em 10 set. 2018.

INEP. Perguntas frequentes. 2019. Disponível em: http://portal.inep.gov.br/educacaobasica/saeb/perguntas-frequentes. Acesso em 10 nov. 2019.

CASTRO, M. H. G. Sistema Nacionais de Avaliação e de informações educacionais. São Paulo em Perspectiva. São Paulo, v. 14, n. 1. p. 121 - 128, 2000.

HORTA NETO, J. L. Um olhar retrospectivo sobre a avaliação externa no Brasil: das primeiras medições em educação até o Saeb de 2005. Revista Iberoamericana de Educación. Madri, v. 42, n. 5. p. 1 - 13, 2007.

KLEIN, R. Utilização da teoria de resposta ao item no sistema nacional de avaliação da educação básica (Saeb). Revista Meta: avaliação. Rio de Janeiro, v. 1, n. 2. p. 125 - 140, mai./ago. 2009.

KLEIN, R. Como está a educação no Brasil? O que fazer? Ensaio: Avaliação e políticas públicas em educação. Rio de Janeiro, v. 14, n. 51. p. 139 - 172, 2006.

SANTOS, A. L. Uma análise do desempenho matemático dos estudantes na rede estadual de ensino no sistema de avaliação da educação básica. In: ENCONTRO REGIONAL DE MA- 
TEMÁTICA APLICADA E COMPUTACIONAL, 6, 2019, Bauru, Caderno de trabalhos completos e resumos [...]. Bauru: Unesp, Faculdade de Ciências, 2019. P. 394 - 400. Disponível em: https://www.fc.unesp.br/\#!/departamentos/matematica/eventos2341/ermac2019/caderno-de-trabalhos-e-resumos/ . Acesso em: 10 nov. 2019.

SANTOS, J. B. P.; TOLENTINO-NETO, L. C. B. O que os dados do Saeb nos dizem sobre o desempenho dos estudantes em matemática? Educação Matemática Pesquisa. São Paulo, v. 17, n. 2. p. $309-333,2015$. 